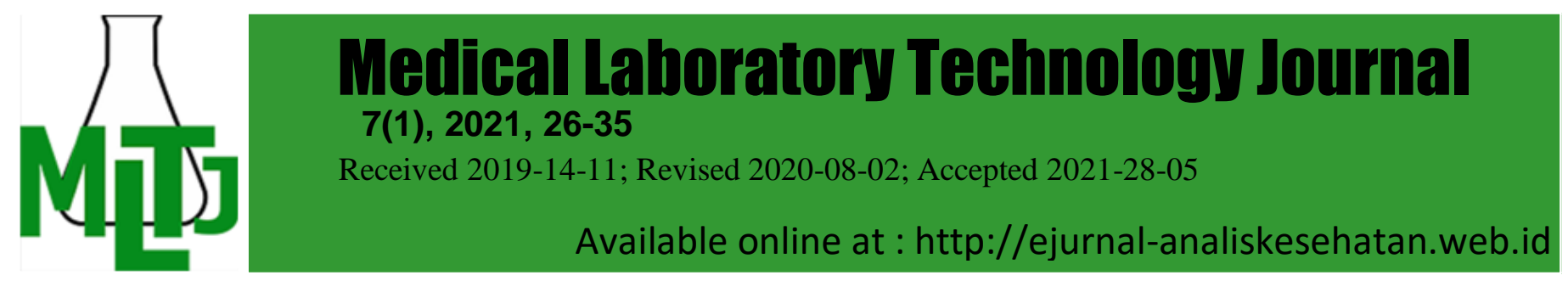

\title{
Uric Acid and P-Wave Dispersion in Subjects with Heart Failure
}

\author{
${ }^{*}$ Recep Kurt', Anıl Şahin ${ }^{2}$ \\ ${ }^{1}$ Sivas Numune Hospital, Department of Cardiology, Sivas, Turkey. ${ }^{2}$ Antalya Training \\ and Research Hospital,Department of Cardiology,Antalya,Turkey. \\ *Email: dr.recepkurt@hotmail.com \\ DOI: $10.31964 / \mathrm{mltj} . v 7 i 1.259$
}

\begin{abstract}
Uric acid used as a marker of cardiovascular risk, which is associated with oxidative stress and inflammation. P-wave dispersion (PWD) is an electrocardiographic measure, which shows heterogeneity of atrial depolarization. It has demonstrated that there is an association of increased PWD with atrial fibrillation. This article's goal was to investigate the relationship between PWD and uric acid in subjects with heart failure (HF). Methods This descriptive, cross-sectional study included a total of 315 stable HF outpatients. The subjects were classified into two groups by their PWD: the normal PWD group consisted of subjects with a PWD lower than $40 \mathrm{~ms}(\mathrm{n}=201)$, and the increased PWD group consisted of subjects with a PWD higher than or equal to $40 \mathrm{~ms}$ (increased PWD) $(n=114)$. Results Significantly higher uric acid levels determined in the increased PWD group, in comparison with the normal PWD group $(7.4 \pm 1.6 \mathrm{mg} / \mathrm{dL}$, vs. $6.5 \pm 1.6 \mathrm{mg} / \mathrm{dL} p<0.001)$. Univariate analyses revealed an association between uric acid, blood urea nitrogen, systolic pulmonary artery pressure, left atrial diameter, and increased PWD. In multivariate logistic regression analysis, there was an association of uric acid level (OR: 1.293; 95\% Cl: 1.106-1.511, p: 0.001), systolic pulmonary artery pressure (OR: $1.027 ; 95 \% \mathrm{Cl}: 1.011$ 1.044, p: 0.001), and left atrial diameter (OR: $1.754 ; 95 \% \mathrm{Cl}: 1.028-2.992$, p: 0.039 ) with PWD. The receiver operating characteristics (ROC) curve analysis demonstrated that the optimal cut-off level of uric acid for predicting increased PWD was $\geq 7.1 \mathrm{mg} / \mathrm{dL}$ with a specificity of 77 percent and a sensitivity of 81 percent. Conclusion Our study results suggest that serum uric acid levels independently correlated with PWD in subjects with HF.
\end{abstract}

Keywords: P-wave dispersion; uric acid; heart failure

\section{INTRODUCTION}

Heart failure (HF) represents a complex clinical syndrome, which is related to high mortality and morbidity rates (Bui et al, 2011). P-wave dispersion (PWD) described as the difference between the longest and shortest $P$-wave durations on 12-lead electrocardiography (ECG). Furthermore, PWD is a non-invasive marker of heterogeneity of atrial depolarization (Aizawa et al, 2017). Several studies have shown that increased PWD can predict atrial fibrillation (AF) (Pérez-Riera et al, 2016). $\mathrm{AF}$ and HF commonly coexist. When they exist in conjunction, each condition has a more severe course with an increased risk of mortality (Carlisleet al, 2019). Previous studies have demonstrated that oxidative stress and inflammation can play a significant part in the development of AF in patients with HF (Oikonomou et al, 2019).

Uric acid, which represents the final product of purine metabolism, is a surrogate marker of inflammation and oxidative stress (Bergamini et al, 2009). The xanthine oxidase enzyme mediates its production. A correlation of increased levels of uric acid with oxidative stress, endothelial dysfunction, and cardiovascular risk has Corresponding Author: Recep Kurt Department of Cardiology Sivas Numune Hospital, Turkey. Email: dr.recepkurt@hotmail.com 
revealed (Borghi et al, 2018; Shao et al, 2019; Zhao et al, 2012). Nevertheless, a relationship of increased uric acid levels with the development of AF has shown in a few studies. Best of our knowledge, the relationship between uric acid levels and PWD, which is a predictor of AF, has not been studied in subjects with HF in the literature. This article's goal was to examine the relationship between PWD and uric acid levels in HF subjects.

\section{MATERIALS AND METHOD Patients and Methods}

This descriptive, cross-sectional research involved a total of 315 (142 females and 173 males) outpatients with the New York Heart Association (NYHA) functional class II, III, and NYHA ambulatory functional Class IV HF. Left ventricular systolic dysfunction (a left ventricular ejection fraction of $<50$ percent) determined in all of the subjects. 12-lead ECG and blood samples obtained on the same day. Patients with thyroid dysfunction, gout, infectious diseases, atrial fibrillation, connective tissue diseases, neoplastic processes, neurologic disorders, end-stage renal disease, and patients taking drugs, which could influence uric acid metabolism (except for diuretics), were not included in the research.

Written informed consent received from every subject. The Ethics Committee of Cumhuriyet University (Sivas, Turkey) approved the study protocol. The research carried following the principles of the Declaration of Helsinki.

\section{ECG examination}

Twelve-lead ECG acquired following a 10-min rest with a $20 \mathrm{~mm} / \mathrm{Mv}$ amplitude and $50 \mathrm{~mm} / \mathrm{sec}$ rate with the standard lead positions. The ECG readings were evaluated by two cardiologists, blinded to the patient data. P-wave duration measurement performed in all leads from the start of the P-wave, which defined as the point at the initial deflection of the $\mathrm{P}$-wave crossed the isoelectric line, to the ending of the P-wave, which defined as the point at the final deflection of P-wave crossed an isoelectric line. Increased PWD defined as a PWD of $\geq 40$ ms(Dilaveris, 1998). Increased PWD is associated with AF. Patients in the current research classified into two groups based on their PWD: the normal PWD group with a PWD $<40$ ms $(n=201)$ and the increased PWD group with a PWD $\geq 40$ ms $(n=$ 114).

\section{Transthoracic echocardiography examination}

All patients underwent transthoracic echocardiography (TTE). TTE examinations performed using a $2.5 \mathrm{MHz}$ transducer and a Vivid 7 Dimension® (GE Vingmed Ultrasound AS, N-3190 Horten, Norway) echocardiography device. The measurements carried out in a left-side decubitus position following a rest time of min through the standard parasternal long axis, short axis, and apical four- and fivechamber view windows. The measurements of the interventricular septum thickness, left ventricular posterior wall thickness, left ventricle (LV) end-systolic diameter, and LV end-diastolic diameter was made just below the mitral valve in the parasternal long-axis view using M-mode examination. In the parasternal long-axis view, M-mode examination used to measure the left atrium size. The left ventricular ejection fraction computed using the biplane Simpson's method from apical four-chamber and twochamber views following the recommendation of the American Society of Echocardiography (Lang RM et al., 2005). The calculation of the systolic pulmonary artery pressure (SPAP) was performed through the peak velocity of tricuspid regurgitation and estimated right atrial pressure (Lancelotti et al, 2010).

significant statistically 


\section{RESULTS AND DISCUSSION}

Table 1. Baseline Characteristics of the Patients

\begin{tabular}{|c|c|c|c|c|}
\hline Variables & $\begin{array}{l}\text { All } \\
\text { patients } \\
(n=315)\end{array}$ & $\begin{array}{l}\text { Group } 1 \\
\text { (PWD<40ms) } \\
(n=201)\end{array}$ & $\begin{array}{l}\text { Group } 2 \\
\text { (PWD } \geq 40 \mathrm{~ms}) \\
(\mathrm{n}=114)\end{array}$ & p-value \\
\hline \multicolumn{5}{|c|}{ Baseline charecteristics } \\
\hline Age (yr) & $65 \pm 12$ & $65 \pm 13$ & $66 \pm 12$ & 0,486 \\
\hline Female & $142(\% 45)$ & $97(\% 48)$ & $45(\% 40)$ & 0,132 \\
\hline Hypertension & $190(\% 60)$ & $122(\% 61)$ & $68(\% 60)$ & 0,855 \\
\hline Diabetes mellitus & $128(\% 41)$ & $87(\% 43)$ & $41(\% 36)$ & 0,204 \\
\hline $\mathrm{CAD}(\%)$ & $224(\% 71)$ & $140(\% 70)$ & $84(\% 74)$ & 0,440 \\
\hline BMI $\left(\mathrm{kg} / \mathrm{m}^{2}\right)$ & $27 \pm 4,0$ & $27 \pm 3,7$ & $27 \pm 4,4$ & 0,282 \\
\hline ACE/ARB(\%) & $271(\% 86)$ & $173(\% 86)$ & $98(\% 86)$ & 1,000 \\
\hline Beta blocker(\%) & $284(\% 90)$ & $181(\% 90)$ & $103(\% 90)$ & 1,000 \\
\hline Thiazides(\%) & $124(\% 39)$ & $83(\% 41)$ & $41(\% 36)$ & 0,352 \\
\hline Loop diuretics(\%) & $205(\% 65)$ & $131(\% 65)$ & $74(\% 65)$ & 1,000 \\
\hline $\begin{array}{l}\text { Aldosterone } \\
\text { anagonists(\%) }\end{array}$ & $210(\% 67)$ & $131(\% 65)$ & $79(\% 69)$ & 0,456 \\
\hline \multicolumn{5}{|c|}{ Echocardiographic findings } \\
\hline LVEF (\%) & $33 \pm 6$ & $33 \pm 6$ & $33 \pm 7$ & 0,753 \\
\hline $\mathrm{LA}(\mathrm{cm})$ & $4,5 \pm 0,5$ & $4,4 \pm 0,5$ & $4,6 \pm 0,5$ & $<0,001$ \\
\hline LVEDD(cm) & $5,6 \pm 0,5$ & $5,5 \pm 0,6$ & $5,6 \pm 0,6$ & 0,571 \\
\hline SPAP (mmhg) & $38 \pm 17$ & $34 \pm 16$ & $43 \pm 16$ & $<0,001$ \\
\hline \multicolumn{5}{|l|}{ Laboratory findings } \\
\hline Hemoglobin (g/dL) & $12,5 \pm 2,0$ & $12,5 \pm 2,0$ & $12,5 \pm 2,0$ & 0,649 \\
\hline $\mathrm{BUN}(\mathrm{mg} / \mathrm{dL})$ & $23 \pm 10$ & $21 \pm 10$ & $25 \pm 10$ & 0,004 \\
\hline Creatinine (mg/dL) & $1,0 \pm 0,3$ & $1,0 \pm 0,3$ & $1,0 \pm 0,3$ & 0,092 \\
\hline Sodium (mmol/L) & $136 \pm 4$ & $136 \pm 4$ & $136 \pm 4$ & 0,765 \\
\hline Potassium(mmol/l) & $4,3 \pm 0,5$ & $4,3 \pm 0,5$ & $4,2 \pm 0,5$ & 0,133 \\
\hline WBC cells/ $\mu \mathrm{l}$ & $7859 \pm 2414$ & $7720 \pm 2385$ & $8103 \pm 2457$ & 0,177 \\
\hline Platelet $\left(10^{3} \times\right.$ cells $\left./ \mu \mathrm{l}\right)$ & $255 \pm 120$ & $255 \pm 124$ & $255 \pm 114$ & 0,998 \\
\hline Uric acid $(\mathrm{mg} / \mathrm{dl})$ & $6,8 \pm 1,6$ & $6,5 \pm 1,6$ & $7,4 \pm 1,6$ & $<0,001$ \\
\hline
\end{tabular}

Abbreviations: PWD, P wave dispersion; DM, diabetes mellitus; CAD, coronary artery disease; BMI, body mass index; $A C E$, angiotensin-converting enzyme; ARB, angiotensin receptor blocker; LVEF, left ventricle ejection fraction; LA, left atrial diameter; SPAP, systolic pulmonary artery pressure; LVEDD, left ventricle enddiastolic diameter, BUN, blood urea nitrogen; WBC, white blood cell.

Three hundred fifteen subjects in total (142 females and 173 males) with an average age of $65 \pm 12$ years enrolled in the present research. The mean body mass index (BMI) of the patients was $27 \pm 4.0 \mathrm{~kg} / \mathrm{m}^{2}$. The BMl values were similar among the males and females in this study. Comorbidities of the study population included hypertension $(60 \%, n=190)$, diabetes mellitus (DM) $(41 \%, n=128)$, and coronary artery disease (CAD) $(71 \%, n=224)$. The mean ejection fraction (EF) was $33 \pm 6 \%$. Significantly higher uric acid levels determined in the increased PWD group in comparison with the normal PWD group $(7.4 \pm 1.6 \mathrm{mg} / \mathrm{dL}$, vs. $6.5 \pm 1.6 \mathrm{mg} / \mathrm{dL} p<$ 0.001 ). The left atrial diameter (LA) and systolic pulmonary artery pressure (SPAP) was also significantly higher ( $4.6 \pm 0.5$ vs. $4.4 \pm 0.5, p<0.001$ and $43 \pm 16$ vs. $34 \pm$ 
16, $p<0.001$, respectively) in the increased PWD group, in comparison with the normal PWD group. Baseline characteristics, echocardiographic parameters, and laboratory data presented in Table 1.

Table 2. Spearman Correlation Coefficients for PWD

\begin{tabular}{lll}
\hline Variable & $\begin{array}{l}\text { PWD } \\
\text { level }\end{array}$ & $\begin{array}{l}\text { p- } \\
\text { value }\end{array}$ \\
\hline LA & 0,311 & $<0,001$ \\
LVEDD & 0,136 & 0,016 \\
SPAP & 0,266 & $<0,001$ \\
BUN & 0,295 & $<0,001$ \\
Creatinin & 0,228 & $<0,001$ \\
Uric acid & 0,353 & $<0,001$ \\
\hline
\end{tabular}

Abbreviations:PWD, $P$ wave dispersion; SPAP, systolic pulmonary artery pressure; LA, left atrial diameter; LVEDD, left ventricule enddiastolic diameter BUN, blood ure nitrogen

Table 3. Univariate and Multivariate Analyses for Predicting Increased PWD

\begin{tabular}{|c|c|c|c|c|c|c|}
\hline \multirow[b]{2}{*}{ Variable } & \multicolumn{3}{|c|}{$\underline{\text { Univariate }}$} & \multicolumn{3}{|c|}{ Multivariate } \\
\hline & $\bar{p}$ & OR & $(95 \% \mathrm{Cl})$ & $\bar{P}$ & OR & $(95 \% \mathrm{Cl})$ \\
\hline \multicolumn{7}{|c|}{ Statistically significant variables } \\
\hline Uric Acid & $<0,001$ & 1,374 & $1,185-1,593$ & 0,001 & 1,293 & $1,106-1,511$ \\
\hline SPAP & $<0,001$ & 1,035 & $1,020-1,051$ & 0,001 & 1,027 & $1,011-1,044$ \\
\hline LA & $<0,001$ & 2,859 & $1,747-4,679$ & 0,039 & 1,754 & $1,028-2,992$ \\
\hline BUN & 0,004 & 1,035 & $1,011-1,060$ & & & \\
\hline \multicolumn{7}{|c|}{ Variables correlating with increased PWD } \\
\hline Creatinin & 0,086 & 1,812 & $0,919-3,573$ & & & \\
\hline LVEDD & 0,569 & 1,113 & $0,760-1,649$ & & & \\
\hline
\end{tabular}

All the variables from Table 1 were examined and only those significant at $P<0.05$ level and correlated with PWD are shown in univarite analysis. Multivariate logistic regression analysis including all the variables inunivariate analysis with forward wald method. Cl: Confidence interval; OR: odds ratio, PWD, P wave dispersion; SPAP, systolic pulmonary artery pressure; LA,left atrial diameter ; BUN, blood urea nitrogen; LVEDD, left ventriculeenddiastolic diameter 


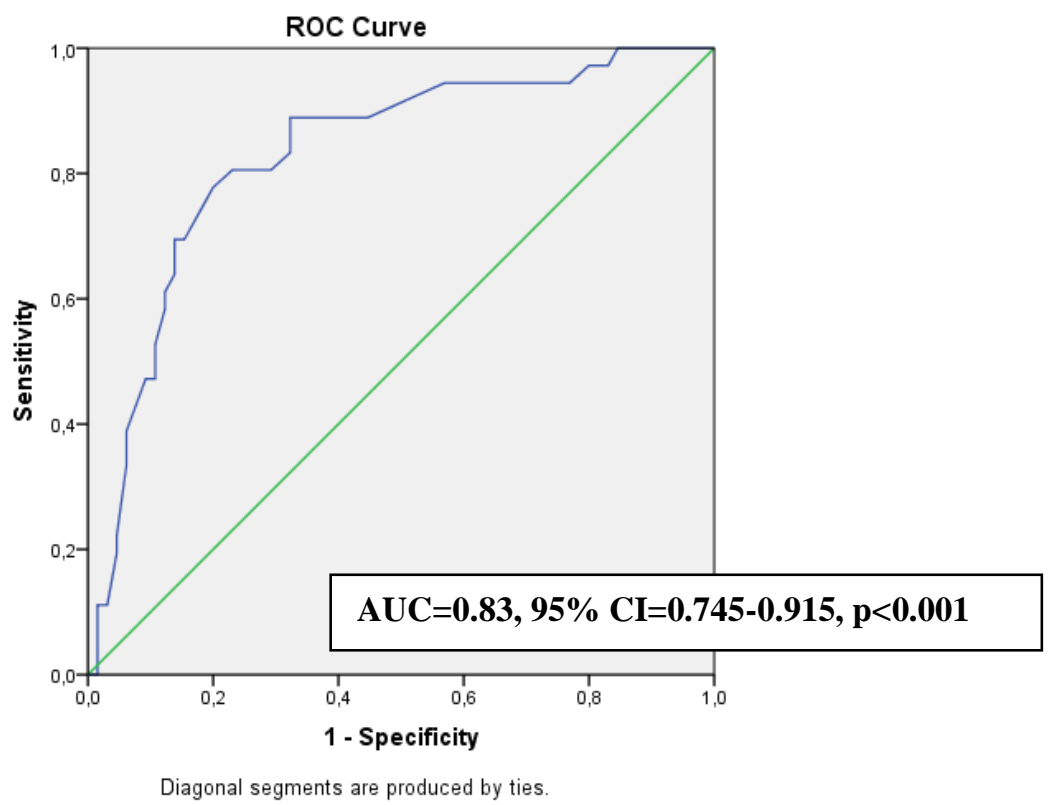

Figure 1. ROC Curve of Uric Acid to Predict Increased PWD

As presented in Table 2, uric acid, blood urea nitrogen (BUN), creatinine, left atrial diameter, SPAP, and left ventricular end-diastolic diameter positively correlated with PWD. Table 3 presents the findings of the univariate and multivariate logistic regression analyses for increased PWD. Uric acid, SPAP, left atrial diameter, and BUN was associated with increased PWD in univariate analyses. In the multivariate logistic regression analysis, uric acid (OR: 1.293; 95\% Cl: 1.106-1.511, p: 0.001), SPAP (OR: 1.027; 95\% Cl: 1.011-1.044, p: 0.001), and left atrial diameter (OR: $1.754 ; 95 \% \mathrm{Cl}: 1.028-2.992$, p: 0.039 ) remained correlated with increased PWD after adjusting for the variables that significantly correlated with PWD in the univariate analyses.

The ROC curve analysis indicated that the optimal cut-off level of uric acid for predicting increased PWD was $\geq 7.1 \mathrm{mg} / \mathrm{dl}$ with a specificity of $77 \%$ and sensitivity of $81 \%(\mathrm{AUC}=0.83,95 \% \mathrm{Cl}=0.745-0.915, \mathrm{p}<0.001$, Figure 1$)$.

As far as we know, the current research represents the first study in the literature, which shows an independent correlation between PWD and serum uric acid levels in subjects with HF.

Similar pathophysiological pathways are involved in $\mathrm{HF}$ and $\mathrm{AF}$, and the concomitance of these two conditions is associated with mortality (Kotecha et al, 2015). Oxidative stress and inflammation play a significant part in the development of $\mathrm{AF}$ in subjects with HF (Zacharia et al, 2019). Uric acid, which is the final product of purine metabolism, represents an important marker of inflammation and oxidative stress. Several studies have suggested a relationship between high uric acid levels and the development of AF (Tamariz et al, 2011; Nyrnes et al, 2014).

$\mathrm{P}$-wave dispersion, which described as the difference between the longest and shortest P-wave duration on 12-lead ECG, represents a non-invasive marker of heterogeneity of atrial depolarization. The increased dispersion of atrial refractoriness has shown to induce reentry, leading to AF(Pérez-Riera et al, 2016). A PWD of 40 ms or higher defined as increased PWD. Dilaveris et al. reported that a PWD value of $40 \mathrm{~ms}$ predicted AF with a sensitivity of $83 \%$, a specificity of $85 \%$, and a positive predictive value of $89 \%$ (Dilaveris et al,1998). 
Increased PWD has associated with certain cardiovascular disorders including cardiomyopathies, rheumatic mitral valve stenosis, stroke and hypertension all of which correlated with a high risk of AF (Dogan et al, 2012; Kocaoglu et al, 2012; Russo et al, 2017; Tsioufis et al, 2019). The current study may indicate that the association between uric acid and the development of AF can be due to increased PWD.

Several studies indicated a correlation between uric acid and PWD under certain clinical conditions. Su et al. investigated the association between PWD and the maximum $\mathrm{P}$-wave duration and rapid deterioration in kidney functions and found an independent association between high uric acid levels and increased PWD ( $\beta=$ $0.281, p=0.002$ ) (Su et al, 2012). Çakar et al. stated that serum uric acid levels were associated with minimum, maximum, and mean $\mathrm{P}$-wave durations in military jet pilots and transport aircraft aircrew $(r=0.355, p=0.002 ; r=0.318, p=0.006$; and $r=0.422$, $p<0.001$, respectively) (Çakar et al, 2016).

The association between uric acid and PWD may be due to the relationship between uric acid and the selective increase in atrial oxidative stress. Uric acid is a marker of upregulated xanthine oxidase activity, which leads to the production of reactive oxygen species, which induce atrial tissue injury (Bergamini et al,2009). Furthermore, uric acid production due to microvascular tissue hypoxia may affect the atrial tissue via systemic oxidative stress. Increased xanthine oxidase activity may lead to cardiac dysfunction and the progression of HF by inducing oxidative stress and uric acid production (Borghi et al, 2019; Huang et al, 2019). Several studies have shown that lowering uric acid levels without altering xanthine oxidase activity does not provide clinical benefit (George et al,2006; Ogino et al, 2010). Therefore, xanthine oxidase activity appears to play a significant part in oxidative stress. Depression of cardiac functions and increased oxidative stress may be responsible for atrial fibrosis, which may be associated with increased PWD. Moreover, increased uric acid levels may increase PWD by inducing endothelial dysfunction and activating the reninangiotensin-aldosterone system (Johnson et al, 2005).

In the present study, SPAP found to be significantly associated with increased PWD. Similarly, Guntekin et al. detected a positive association between SPAP and PWD in patients with mitral valve stenosis $(r=0.295, P=0.047)$ (Guntekin et al, 2008). However, in the study by Turhan et al., the decrease in SPAP after mitral balloon valvuloplasty was not found to be associated with a parallel decrease in PWD (Turhan et al, 2002). Based on these findings, we suggest that the relation between SPAP and PWD takes place, as diastolic dysfunction in HF may lead to an increase in pulmonary artery pressure in addition to atrial dilatation and fibrosis. The other possible explanation is that enhanced sympathetic activity in patients with HF may increase both PWD and SPAP to a similar extent (Akutsu et al, 2013; Tukek et al, 2000).

In the present research, the left atrial diameter found to be significantly associated with increased PWD. Some studies have also found similar associations, while some authors have not shown such an association (Ozyigit et al, 2016; Sarvari et al, 2016; Koide et al, 2002; Tukek et al, 2001). P-wave dispersion may be affected by the delay in site-dependent intra-atrial conduction before the onset of left atrial dilatation (Hatam et al, 2014; Gazi et al, 2015). However, increased sympathetic activity may affect the propagation of the sinus impulse in subjects with HF. It has also reported that PWD may be affected by increased left atrial pressure, diastolic changes, and intra atrial oxidative stress (Gudul et al, 2017).

In the current study, BUN and creatinine levels positively correlated with PWD. 
However, neither BUN nor creatinine was an independent predictor of increased PWD in multivariate regression analysis. Another study showed an independent association between PWD and deterioration in kidney functions (Su et al, 2012). The association between PWD and kidney functions may be due to subclinical inflammation, endothelial dysfunction, systemic neurohormonal activation, and oxidative stress in patients with kidney disease.

Although the clinical implementation of our findings is a little bit difficult and dubious, the increased levels of uric acid, as an inflammatory biomarker in heart failure, might corrupt the atrial conduction. Our data analysis showed that increased levels of uric acid could be a trigger for atrial arrhythmias. Furthermore, large-scale studies evaluating the impact of drugs interacting with uric acid metabolism on arrhythmias can enlighten this association.

Nonetheless, there are a few limitations of the present research. Firstly, this study has a small sample size. Secondly, we used the manual measurement of Pwaves by a magnifying lens rather than a computer-assisted calculation. In previous studies, digital and signal-averaging ECG systems utilized to evaluate PWD more accurately (Tukek et al, 2000; Dilaveris et al, 1999). Thirdly, the current research represents a cross-sectional study and further longitudinal studies required for confirming the relationship between PWD and uric acid. Another limitation was the lack of regular ambulatory ECG monitorization. That is why paroxysmal AF attacks could not be recorded. Finally, it is possible that the left atrial maximal diameter, measured in the present research, does not represent the left atrial size and volume in subjects with HF accurately.

\section{CONCLUSION}

In conclusion, our study results suggest an independent association between serum uric acid levels and PWD in subjects with HF. The relationship between increased uric acid levels and the development of AF in subjects with HF can originate from increased PWD. Furthermore, prospective research with larger sample sizes required for confirming this association and demonstrating underlying pathophysiological mechanisms. Such studies would also provide information to prevent AF in patients with HF.

\section{ACKNOWLEDGEMENT}

The authors would like to thank all parties involved in this study.

\section{CONFLICT OF INTEREST}

The authors declare that there are no conflicts of interest regarding the publication of this paper.

\section{REFERENCES}

Aizawa, Y., Watanabe, H., \& Okumura, K. (2017). Electrocardiogram (ECG) for the prediction of incident atrial fibrillation: an overview. Journal of atrial fibrillation, 10(4).

Akutsu, Y., Kaneko, K., Kodama, Y., Miyoshi, F., Li, H. L., Watanabe, N., ... \& Kobayashi, Y. (2013). A combination of $P$ wave electrocardiography and plasma brain natriuretic peptide level for predicting the progression to persistent atrial fibrillation: comparisons of sympathetic activity and left atrial size. Journal of Interventional Cardiac Electrophysiology, 38(2), 79-84.

Bergamini, C., Cicoira, M., Rossi, A., \& Vassanelli, C. (2009). Oxidative stress and 
hyperuricaemia: pathophysiology, clinical relevance, and therapeutic implications in chronic heart failure. European journal of heart failure, 11(5), 444452.

Borghi, C., Palazzuoli, A., Landolfo, M., \& Cosentino, E. (2020). Hyperuricemia: a novel old disorder-relationship and potential mechanisms in heart failure. Heart failure reviews, 25(1), 43-51.

Borghi, C., Tykarski, A., Widecka, K., Filipiak, K. J., Domienik-Karłowicz, J., KostkaJeziorny, K., ... \& Mancia, G. (2018). Expert consensus for the diagnosis and treatment of patient with hyperuricemia and high cardiovascular risk. Cardiology journal, 25(5), 545-564.

Bui, A. L., Horwich, T. B., \& Fonarow, G. C. (2011). Epidemiology and risk profile of heart failure. Nature Reviews Cardiology, 8(1), 30.

Carlisle, M. A., Fudim, M., DeVore, A. D., \& Piccini, J. P. (2019). Heart failure and atrial fibrillation, like fire and fury. JACC: Heart Failure, 7(6), 447-456.

Çakar, M., Metin, S., Balta, S., Öztürk, C., Demirkol, S., Çakmak, T., ... \& Sen, A. (2016). Military jet pilots have higher $p$-wave dispersions compared to the transport aircraft aircrew. International journal of occupational medicine and environmental health, 29(4), 563.

Dilaveris, P. E., Gialafos, E. J., Chrissos, D., Andrikopoulos, G. K., Richter, D. J., Lazaki, E., \& Gialafos, J. E. (1999). Detection of hypertensive patients at risk for paroxysmal atrial fibrillation during sinus rhythm by computer-assisted $P$ wave analysis. Journal of hypertension, 17(10), 1463-1470.

Dilaveris, P. E., Gialafos, E. J., Sideris, S. K., Theopistou, A. M., Andrikopoulos, G. K., Kyriakidis, M., ... \& Toutouzas, P. K. (1998). Simple electrocardiographic markers for the prediction of paroxysmal idiopathic atrial fibrillation. American heart journal, 135(5), 733-738.

Dogan, U., Dogan, E. A., Tekinalp, M., Tokgoz, O. S., Aribas, A., Akilli, H., ... \& Yuruten, B. (2012). P-wave dispersion for predicting paroxysmal atrial fibrillation in acute ischemic stroke. International Journal of Medical Sciences, 9(1), 108.

Gazi, E., Gencer, M., Hanci, V., Temiz, A., Altun, B., Barutcu, A., ... \& Colkesen, Y. (2015). Atrial conduction time, and left atrial mechanical and electromechanical functions in patients with polycystic ovary syndrome: interatrial conduction delay: cardiovascular topics. Cardiovascular journal of Africa, 26(6), 217-221.

George, J., Carr, E., Davies, J., Belch, J. J. F., \& Struthers, A. (2006). Clinical perspective. Circulation, 114(23), 2508-2516.

Gudul, N. E., Karabag, T., Sayin, M. R., Bayraktaroglu, T., \& Aydin, M. (2017). Atrial conduction times and left atrial mechanical functions and their relation with diastolic function in prediabetic patients. The Korean journal of internal medicine, 32(2), 286.

Guntekin, U., Gunes, Y., Tuncer, M., Gunes, A., Sahin, M., \& Simsek, H. (2008). Longterm follow-up of $\mathrm{P}$-wave duration and dispersion in patients with mitral stenosis. Pacing and clinical electrophysiology, 31(12), 1620-1624.

Hatam, N., Aljalloud, A., Mischke, K., Karfis, E. A., Autschbach, R., Hoffmann, R., \& Goetzenich, A. (2014). Interatrial conduction disturbance in postoperative atrial fibrillation: a comparative study of P-wave dispersion and Doppler myocardial imaging in cardiac surgery. Journal of cardiothoracic surgery, 9(1), 1-9.

Huang, G., Qin, J., Deng, X., Luo, G., Yu, D., Zhang, M., ... \& Wang, L. (2019). Prognostic value of serum uric acid in patients with acute heart failure: A metaanalysis. Medicine, 98(8).

Johnson, R. J., Rodriguez-Iturbe, B., Kang, D. H., Feig, D. I., \& Herrera-Acosta, J. 
(2005). A unifying pathway for essential hypertension. American journal of hypertension, 18(3), 431-440.

Kocaoglu C, Sert A, Aypar E, Oran B, Odabas D, Arslan D, Akin F. (2012). P-wave dispersion in children with acute rheumatic fever. Pediatr Cardiol, 33(1), 90-4.

Koide, Y., Yotsukura, M., Sakata, K., Yoshino, H., \& Ishikawa, K. (2002). Investigation of the predictors of transition to persistent atrial fibrillation in patients with paroxysmal atrial fibrillation. Clinical Cardiology: An International Indexed and Peer-Reviewed Journal for Advances in the Treatment of Cardiovascular Disease, 25(2), 69-75.

Kotecha, D., \& Piccini, J. P. (2015). Atrial fibrillation in heart failure: what should we do?. European heart journal, 36(46), 3250-3257.

Lancellotti, P., Moura, L., Pierard, L. A., Agricola, E., Popescu, B. A., Tribouilloy, C., ... \& Roelandt, J. R. (2010). European Association of Echocardiography recommendations for the assessment of valvular regurgitation. Part 2: mitral and tricuspid regurgitation (native valve disease). European Journal of Echocardiography, 11(4), 307-332.

Lang RM, Bierig M, Devereux RB et al. (2005). Chamber Quantification Writing Group; American Society of Echocardiography's Guidelines and Standards Committee; European Association of Echocardiography. Recommendations for chamber quantification: a report from the American Society of Echocardiography's Guidelines and Standards Committee and the Chamber Quantification Writing Group, developed in conjunction with the European Association of Echocardiography, a branch of the European Society of Cardiology. J Am Soc Echocardiogr, 18, 1440-63.

Nyrnes, A., Toft, I., Njølstad, I., Mathiesen, E. B., Wilsgaard, T., Hansen, J. B., \& Løchen, M. L. (2014). Uric acid is associated with future atrial fibrillation: an 11year follow-up of 6308 men and women-the Tromsø Study. Europace, 16(3), 320-326.

Ogino, K., Kato, M., Furuse, Y., Kinugasa, Y., Ishida, K., Osaki, S., ... \& Doehner, W. (2010). Uric acid-lowering treatment with benzbromarone in patients with heart failure: a double-blind placebo-controlled crossover preliminary study. Circulation: Heart Failure, 3(1), 73-81.

Oikonomou, E., Zografos, T., Papamikroulis, G. A., Siasos, G., Vogiatzi, G., Theofilis, P., ... \& Tousoulis, D. (2019). Biomarkers in atrial fibrillation and heart failure. Current medicinal chemistry, 26(5), 873-887.

Ozyigit, T., Kocas, O., Karadag, B., \& Ozben, B. (2016). Three dimensional left atrial volume index is correlated with $\mathrm{P}$ wave dispersion in elderly patients with sinus rhythm. Wiener klinische Wochenschrift, 128(5-6), 182-186.

Pérez-Riera, A. R., de Abreu, L. C., Barbosa-Barros, R., Grindler, J., FernandesCardoso, A., \& Baranchuk, A. (2016). P-wave dispersion: an update. Indian Pacing and Electrophysiology Journal, 16(4), 126-133.

Sarvari, S. I., Haugaa, K. H., Stokke, T. M., Ansari, H. Z., Leren, I. S., Hegbom, F., ... \& Edvardsen, T. (2016). Strain echocardiographic assessment of left atrial function predicts recurrence of atrial fibrillation. European Heart JournalCardiovascular Imaging, 17(6), 660-667.

Shao, Y., Shao, H., Sawhney, M. S., \& Shi, L. (2019). Serum uric acid as a risk factor of all-cause mortality and cardiovascular events among type 2 diabetes population: Meta-analysis of correlational evidence. Journal of Diabetes and its Complications, 33(10), 107409.

Su, H. M., Tsai, W. C., Lin, T. H., Hsu, P. C., Lee, W. H., Lin, M. Y., ... \& Sheu, S. H. 
(2012). $P$ wave dispersion and maximum $P$ wave duration are independently associated with rapid renal function decline. PloS one, 7(8), e42815.

Tamariz, L., Agarwal, S., Soliman, E. Z., Chamberlain, A. M., Prineas, R., Folsom, A. R., ... \& Alonso, A. (2011). Association of serum uric acid with incident atrial fibrillation (from the Atherosclerosis Risk in Communities [ARIC] study). The American journal of cardiology, 108(9), 1272-1276.

Tsioufis, C., Konstantinidis, D., Nikolakopoulos, I., Vemmou, E., Kalos, T., Georgiopoulos, G., ... \& Tousoulis, D. (2019). Biomarkers of atrial fibrillation in hypertension. Current medicinal chemistry, 26(5), 888-897.

Tüukek, T., Akkaya, V., Atilgan, D., Demirel, Ş., Özcan, M., Güuven, Ö., \& Korkut, F. (2001). Effect of left atrial size and function on P-wave dispersion: A study in patients with paroxysmal atrial fibrillation. Clinical cardiology, 24(10), 676-680.

Tükek, T., Akkaya, V., Demirel, Ş., Sözen, A. B., Kudat, H., Atılgan, D., ... \& Korkut, F. (2000). Effect of Valsalva maneuver on surface electrocardiographic P-wave dispersion in paroxysmal atrial fibrillation. American Journal of Cardiology, 85(7), 896-899.

Turhan, H., Yetkin, E., Senen, K., Yilmaz, M. B., Ileri, M., Atak, R., ... \& Kutuk, E. (2002). Effects of percutaneous mitral balloon valvuloplasty on P-wave dispersion in patients with mitral stenosis. American Journal of Cardiology, 89(5), 607-609.

Zacharia, E., Papageorgiou, N., loannou, A., Siasos, G., Papaioannou, S., Vavuranakis, M., ... \& Tousoulis, D. (2019). Inflammatory biomarkers in atrial fibrillation. Current medicinal chemistry, 26(5), 837-854.

Zhao, Q. Y., Yu, S. B., Huang, H., Cui, H. Y., Mu, Q. I. N., Huang, T., \& Huang, C. X. (2012). Serum uric acid levels correlate with atrial fibrillation in patients with chronic systolic heart failure. Chinese medical journal, 125(10), 1708-1712. 